\title{
Optical and UV Observations of Supernova Remnants
}

\author{
By ROBERTA. FESEN
}

Dartmouth College, Dept. of Physics and Astronomy, Hanover, NH 03755, USA

\begin{abstract}
Recent observations of the galactic supernova remnants the Crab Nebula, SN 1006, Cas A, and the Cygnus Loop are reviewed. New studies of the Crab Nebula suggest its progenitor may have had appreciable mass loss in the form of a circumstellar disk resulting in both a bipolar expansion and formation of the synchrotron 'bays'. Unusually high proper motion knots near to and possibly directed away from the pulsar also have been reported. In the Cas A remnant, a NE jet of ejecta appears to be a plume of mantle material with expansion velocities up to $12000 \mathrm{~km} \mathrm{~s}^{-1}$ or nearly twice that seen in the main ejecta shell. HST observations of the sdOB star located behind SN 1006 indicate symmetrically expanding Fe II ejecta out to $8100 \mathrm{~km} \mathrm{~s}^{-1}$. Lastly, deep images of the Cygnus Loop reveal emission structures similar to those seen in $2 \mathrm{D}$ \& 3D shocked cloud simulations.
\end{abstract}

Optical research on the properties of galactic supernova remnants (SNRs) continues to yield important new results. Though only a small fraction of the radio catalogued $170+$ galactic SNRs are optically detectable, optical measurements permit one to investigate such SNR properties as chemical abundances relative to hydrogen, expansion velocities, gas densities and temperatures, and ejecta filament morphologies and distribution. With the advent of the International Ultraviolet Explorer (IUE) in 1978 and now the Hubble Space Telescope (HST), UV observations on the brighter and less reddened optical SNRs are possible, substantially adding to our knowledge. While many of the brighter galactic SNRs, like the Crab Nebula and the Cygnus Loop, have been studied for many decades by dozens of researchers, important and sometimes unexpected results based on optical and UV observations have been recently reported on even these well studied objects. Many of these findings have significance not only for SNR research but for SN studies as well since they often provide insights into the nature of the progenitor star or on the explosion dynamics. Below is brief (and rather biased) survey of a small sample of recent optical and UV research on four galactic SNRs: the Crab Nebula, the remnant of SN 1006, Cas A, and the Cygnus Loop.

\section{The Crab Nebula (SN 1054)}

Despite the hundreds of research papers devoted to it and its luminous pulsar, the Crab Nebula remains today an active research target for investigating the physical processes which occur in pulsar driven SNRs. Recent optical and UV investigations underscore this assessment by revealing just how incomplete our understanding really has been on this remarkable object. Curiously, the Crab Nebula's filaments, which should be virtually undiluted by interstellar gas, show a definite overabundance of only one element - helium, with little trace of freshly synthesized heavy elements (Henry \& MacAlpine 1982; Pequignot \& Dennefeld 1983). In 1987, Uomoto \& MacAlpine found that the differences of He content among filaments was not random, but showed instead a He concentration along an east-west band which they referred to as the High Helium Band (HHeB). Soon after, MacAlpine et al. (1989) found a filament expansion anisotropy in the form of a north-south bipolar bubble above and below the HHeB. No cause for the $\mathrm{HHeB}$ or this velocity 'pinching' was proposed. However, new work may point to a 


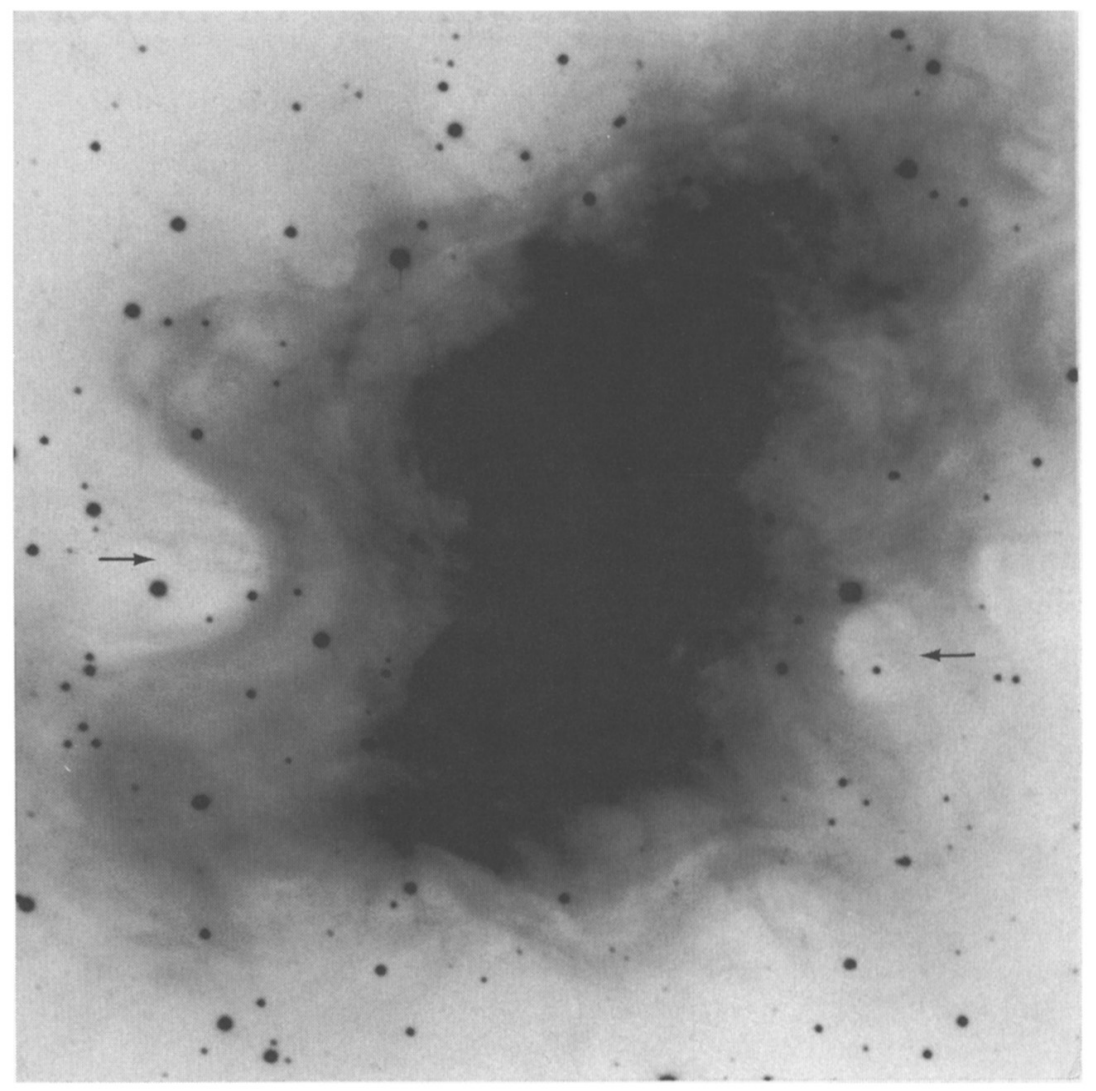

Figure 1. A $8100 \AA$ continuum image of the Crab Nebula showing presence of the East and West indentations or 'bays' in the pulsar's synchrotron emission nebula. North is to the top, east to the left.

possible physical connection between the He-rich filament band, the bipolar expansion, and formation of the remnant's synchrotron emission 'bays'.

Among the remnant's most conspicuous large-scale features on optical images taken with interference-filters which avoid the filaments' emission lines are east and west synchrotron nebula indentations or 'bays' (see Fig. 1). Although often ignored in studies of the remnant, observations indicated that these bays are associated with the remnant's magnetic field. For example, to a high degree of coherence, large radial polarization vectors follow the bays' edges indicating large and very orderly magnetic field loops parallel to bay boundaries (see Michel et al. 1991 and references therein). An optical study of the bays by Fesen, Martin, and Shull (1992: hereafter FMS) found the bays to be old, long lasting remnant features which exhibit proper motions not unlike the brighter central filaments. FMS suggested that the bays are manifestations of a magnetic torus encircling the remnant's center of expansion which effectively blocks the pulsar's relativistic particles thus creating the observed synchrotron emission bays. The E-W bays' rough coincidence with the extent of the $\mathrm{HHeB}$ led FMS to further propose that the magnetic torus is due to a component of the pulsar's magnetic field anchored to a thermal pre-SN disk, whose remains include the HHeB.

While unclear if the $\mathrm{HHeB}$ is composed more of SN ejecta than circumstellar material, 


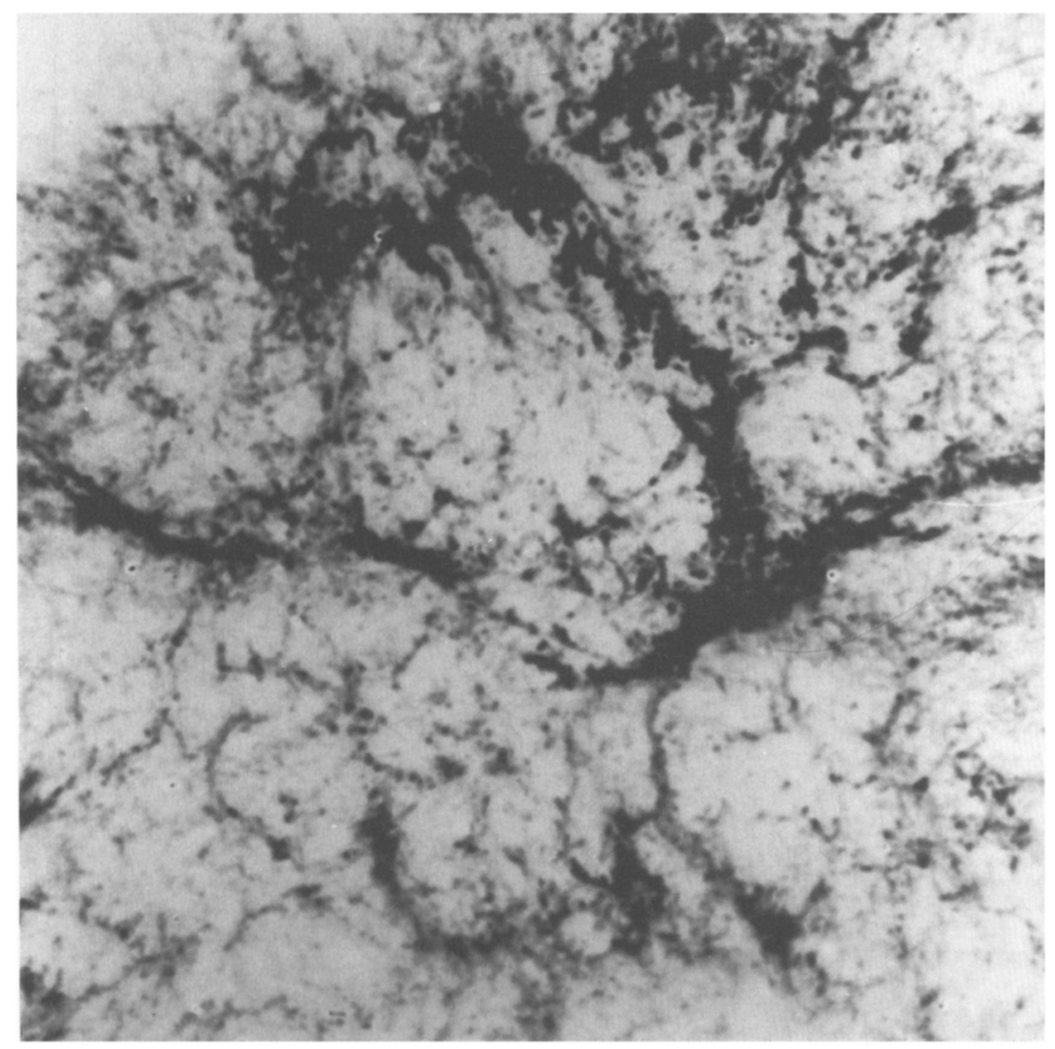

FIgURE 2. $\mathrm{H} \alpha$ image of the middle section of the Crab Nebula illustrating the considerable complexity of the remnant's filamentary structure.

FMS's suggestion of a pre-SN mass loss disk may explain MacAlpine et al.'s (1989) observation of a bipolar expansion. Although optical images such as the $\mathrm{H} \alpha$ image presented in Figure 2 show a highly complex filamentary structure with no apparent symmetry axis, spectroscopic scans do reveal a surprising NS symmetry. A long northsouth slit scanned east-west across the central region of the nebula (see Fig. 3) reveals a well defined shell structure with a sharp inner boundary and a strong velocity pinching near the center of the remnant. This orderly expansion structure means that, contrary to expectations, the nebula's highest expansion velocities are not found towards the center. Combining these data with images that show a virtual absence of filamentary emission in the direction of the east bay (FMS), a picture that emerges is that of a circumstellar disk which both partially blocked the SN's free expansion along an east-west plane and helped focus the pulsar's magnetic field into a EW torus leading to development of the east and west bays. If this model is correct, then it may turn out that the Crab Nebula is somewhat related to SN $1987 \mathrm{~A}$ in the sense that its progenitor also underwent significant mass loss in a disk-like structure.

Recent work on the UV spectra of the Crab filaments has also re-opened the question of significant heavy element abundance variations among filaments. Using the Hopkins Ultraviolet Telescope aboard the ASTRO-1 Space Shuttle mission of December 1990, Blair et al. (1992) examined the nebula's bright concentration of filaments just west of the pulsar. While both blue and redshifted C IV 1550 emission features were detected, 


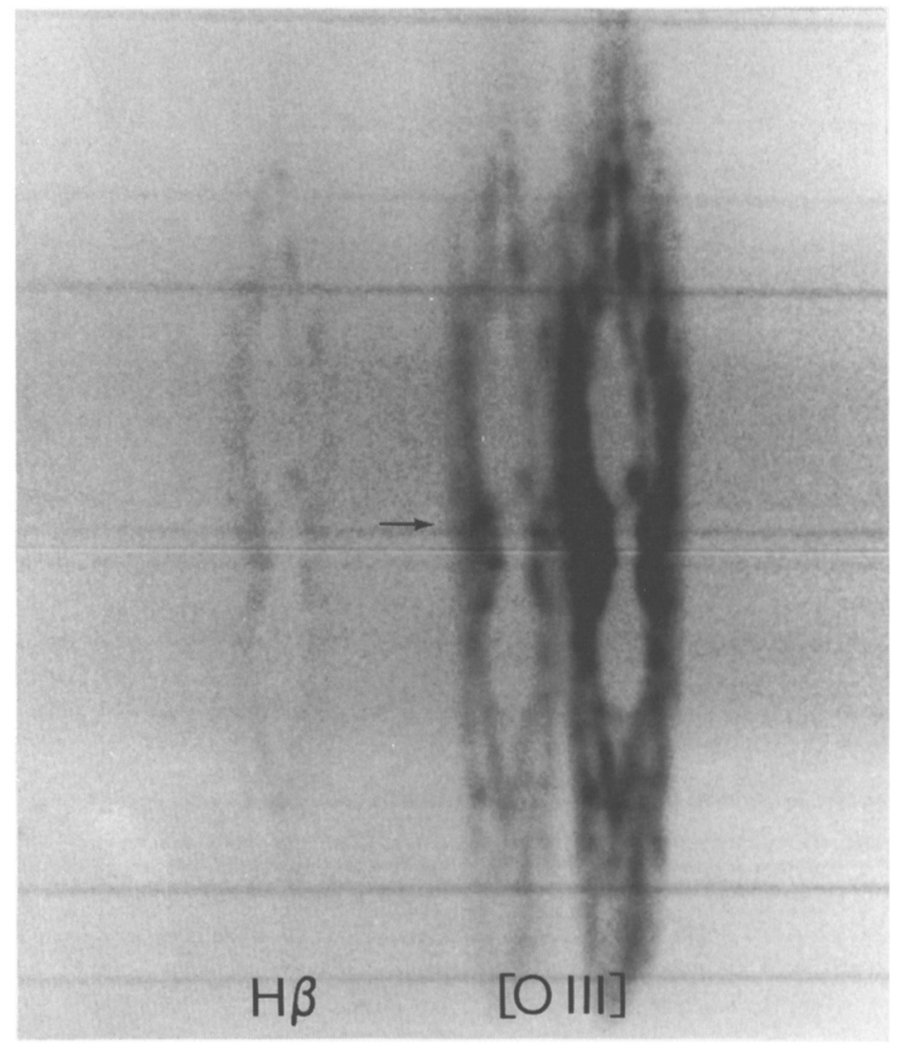

FIgURE 3. Results of a NS slit scan of the Crab's inner section (between the East and West bays) showing the spectral region around $\mathrm{H} \beta$ and [O III] 4959, 5007. Note the strong velocity decrease near the remnant's expansion center (arrow). (From Fesen \& Shull 1993)

only blueshifted He II 1660 emission was seen. This is the first evidence of substantial UV emission line variations in the Crab and may imply large $\mathrm{C} / \mathrm{He}$ abundance differences among filaments in and around the $\mathrm{HHeB}$ region.

Finally, nearly a dozen semi-stellar emission knots have been reported near the Crab pulsar which seem to exhibit remarkable spectral and kinematic properties (MacAlpine et al. 1993). These knots appear roughly aligned in two arcs, one to the north containing seven knots and four to the south. They appear brightest in [O III] emission and have unusually strong [Ar III] 7135 line emission nearly rivaling $\mathrm{H} \alpha$. In addition, proper motion measurements over a two year baseline indicate that while most of these knots have $\mu \leq 0.25^{\prime \prime} \mathrm{yr}^{-1}$, several may have $\mu=0.5^{\prime \prime}-0.8^{\prime \prime} \mathrm{yr}^{-1}$. If these high proper motions are confirmed, these knots would then have the highest velocities known in the remnant, approaching 5000 to $8000 \mathrm{~km} \mathrm{~s}^{-1}$, or more than twice the maximum observed values even in the Crab's northern jet (Fesen \& Staker 1993). The motions appear to be directed away from the pulsar and not the remnant's center of expansion. The nature of these knots is unknown, but they may be related to the pulsar's strong relativistic wind and magnetic field. In any case, these data along with the others described above, make it clear that the Crab Nebula still has things to teach us. 


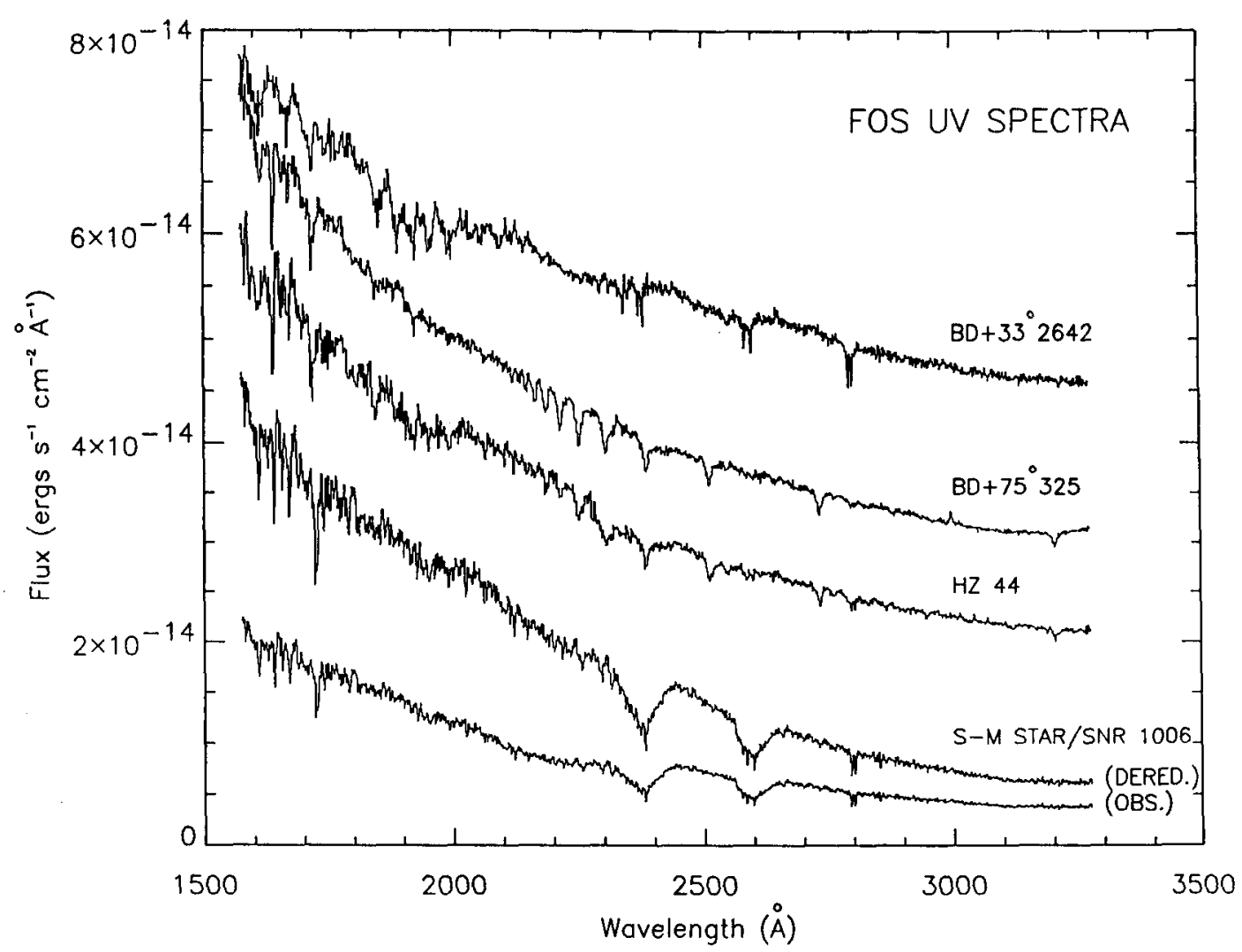

FIgURE 4. HST FOS spectra of the sdOB star behind SN 1006 (bottom two plots) along with FOS spectra of three sdO and early type stars for comparison. (From Wu et al. 1993)

\section{SN 1006 AD}

The galactic SNR G327.6+14.6 is the probable remnant of the bright historical supernova observed in AD 1006. In radio and X-rays, the remnant appears as a limb brightened $30^{\prime}$ diameter shell. Morphological similarities between this SNR and that of Tycho's SNR (SN 1572), SN 1006's high galactic latitude, SN 1006's reported brilliance $(\mathrm{m} \sim-9)$, and a lack of a nearby OB association have all pointed to a likely Type Ia classification. Support for this includes IUE observations of a faint sdOB star fortuitously positioned behind the remnant's center. Low dispersion IUE spectra of this star revealed several strong and broad absorption features including the Fe II resonance lines near 2370 and $2600 \AA$ with an expansion velocity of $5000 \mathrm{~km} \mathrm{~s}^{-1}$, consistent with Type Ia model predictions (Fesen et al. 1988).

Higher resolution and better quality FOS spectra using HST (Fig. 4) greatly clarify SN 1006's induced Fe II absorption features at 2343, 2374, 2382, 2586, and $2599 \AA$ (Wu et al. 1993). After removal of the narrow interstellar absorption lines, the observed line profiles suggest a very symmetric expansion core of Fe II with a FWHM $=8100 \mathrm{~km} \mathrm{~s}^{-1}$ and FWZI $=16600 \mathrm{~km} \mathrm{~s}^{-1}$ (the velocity difference with IUE's measurements is due to significantly better signal-to-noise data in the line wings). This expansion velocity, when combined with the SNR's known age, gives a reverse shock radius $\geq 8.4 \mathrm{pc}$, which in turn with a SNR dia $=30^{\prime}$, implies a distance of $\geq 1.9 \mathrm{kpc}$. Assuming spherical symmetry, 

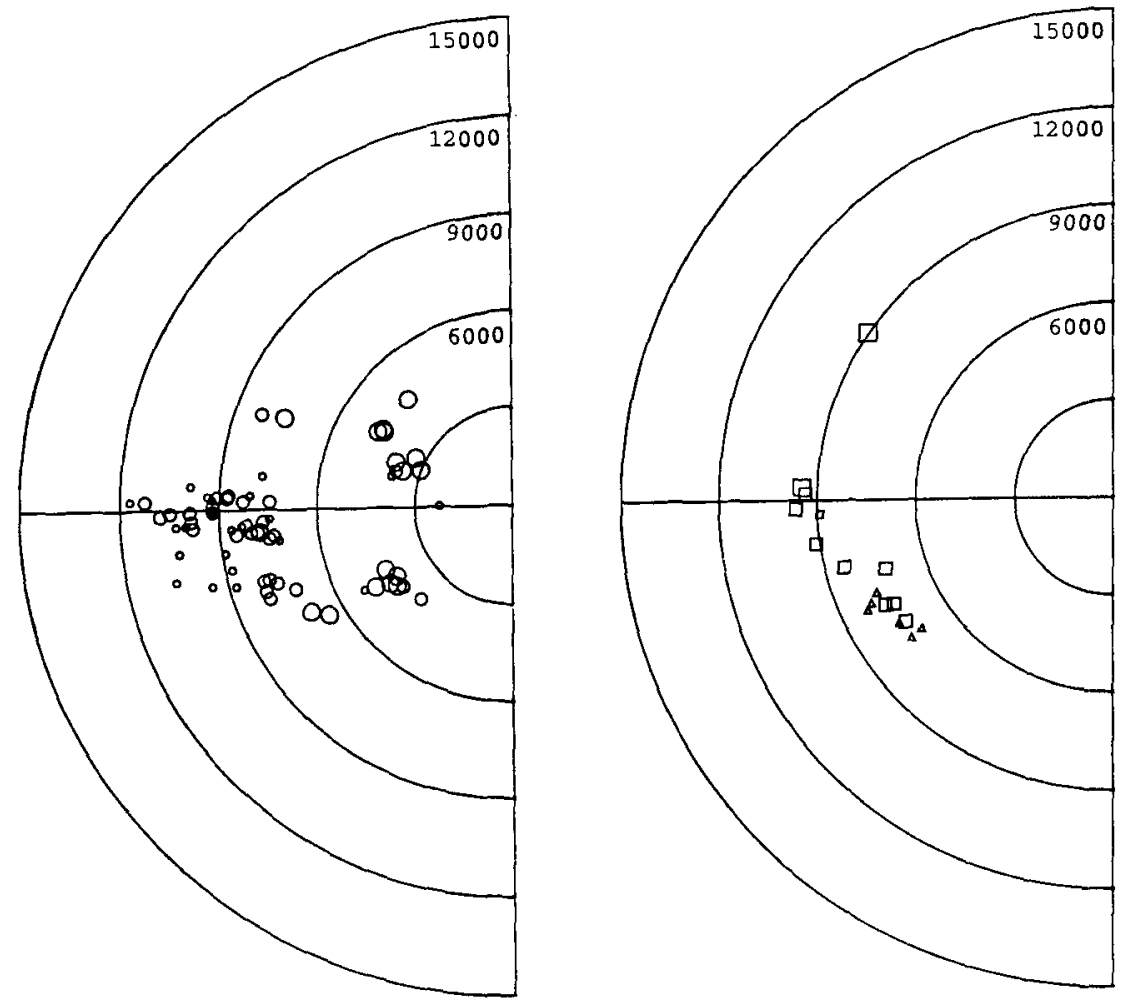

Figure 5. Total space velocities $\left(\mathrm{km} \mathrm{s}^{-1}\right)$ for optical emission knots in and nearby the Cas $A$ jet as viewed from above. Circles indicate position of the FMKs, squares the FMFs, and triangles the 'mixed ejecta knots'. Symbol size indicates relative brightness. (From Gunderson \& Fesen 1993)

the Fe II line profiles indicate a $0.014 \mathrm{M}_{\odot}$ or the equivalent of just a few percent of the total expected Fe mass $\left(0.3-0.7 \mathrm{M}_{\odot}\right)$ from model calculations.

\section{3. $\operatorname{Cas} \mathrm{A}(\mathrm{SN} \approx 1670)$}

Whereas SN 1006 appears to be an example of symmetrical SN expansion, the SNR Cassiopeia A (Cas A) may be a case of a massive star SN (Type Ib/Type II) which underwent a partially asymmetric explosion. Optically, Cas A is a faint nebula of knots and short filaments the majority of which exhibit strong emission lines of $\mathrm{O}, \mathrm{S}, \mathrm{Ar}$, and $\mathrm{Ca}$ but no detectable $\mathrm{H}$ or $\mathrm{He}$ line emissions. The spectral properties of these fast moving knots ('FMKs') are consistent with models for undiluted core ejecta from a massive progenitor star (Chevalier \& Kirshner 1979). In addition to the FMKs, a few dozen slow moving $\mathrm{N}, \mathrm{H}$, and He emitting emission clumps ('QSFs') are seen which appear to be shocked pre-SN circumstellar mass-loss material.

Several years ago, a dozen or so faint outlying knots were found having both highvelocity and $\mathrm{H} \& \mathrm{~N}$ line emission ('FMFs') suggestive of outer N-rich photospheric layer ejecta. A bright knot was later found to have characteristics of both FMKs \& FMFs and thus appeared to represent a possible mixing of outer and inner layers of the star (Fesen 
\& Becker 1991). If true, this chemically mixed knot then also suggests that, at the time of its supernova outburst, the Cas A star still possessed a thin outer layer of $\mathrm{H}+\mathrm{N}$. Interestingly, this mixed knot is located along Cas A's northeastern rim where there is a 'jet' of filaments (mostly FMKs) extending a considerable distance beyond the SNR's bright radio and $\mathrm{X}$-ray emission shell. A mixing of mantle and envelope material might have occurred if the jet represents a plume of underlying material ejected at unusually high velocity.

In order to explore the jet's kinematics and chemical composition with distance from the expansion center, long slit spectra yielding radial velocity and relative line emission strengths for 80 knots were recently obtained and analyzed (Gunderson \& Fesen 1993). These spectra well sampled the jet's three main 'sprays' of filaments, representing the bulk of the jet's optical emission. The spectra, which offer a detailed look at the Cas A jet, reveal several interesting properties. The jet's structure appears quite extensive with over a hundred [S II] $\lambda \lambda 6716,6731$ ejecta knots extending out to $12000 \mathrm{~km} \mathrm{~s}^{-1}$ in expansion velocity - nearly twice that of the remnant's main shell of ejecta. The [S II]/[O I] ratio appears to increase in knots with increasing radial distance, implying a possible S/O abundance gradient along the length of the jet. About a dozen 'mixed ejecta knots' were identified confirming that mixing of the star's original inner and outer layers did occur and is not limited to just one peculiar knot. Both the jet's FMFs and mixed ejecta knots appear within a surprisingly narrow velocity range between 7000 and $9500 \mathrm{~km} \mathrm{~s}^{-1}$. In summary, a highly asymmetric explosion in this section of the Cas A $\mathrm{SNR}$ is suggested which may have relevance in some asymmetrical line emissions observed in extragalactic SNe.

\section{The Cygnus Loop SNR}

High velocity intercloud shocks present in older supernova remnants $\left(\mathrm{V}=10^{2}-10^{3}\right.$ $\mathrm{km} \mathrm{s}^{-1}$ ) can often be detected optically as faint filamentary $\mathrm{H} \alpha$ emission along the SNR's limb if the shock moves through a partially neutral medium. These intercloud shocks exhibit a hydrogen Balmer dominated spectrum and are often referred to as Balmerdominated or nonradiative shock filaments. Both radiative and nonradiative filaments mark locations where portions of the shock front are viewed tangentially (the 'wavy sheet' model; Hester 1987). The Cygnus Loop is a relatively nearby $(0.75 \mathrm{kpc})$, bright, low obscuration $[E(B-V)=0.08$ ] SNR making it an excellent subject for detailed spatial studies of SNR shock emissions. Wide-field Schmidt $\mathrm{H} \alpha$ images of portions of the Cygnus Loop (Fesen, Kwitter, \& Downes 1992) found many of the 2D and 3D numerical shock cloud simulation predictions of Klein et al. (1991) and Stone \& Norman (1992) including cloud deformation, gas stripping, shock reflection and diffraction around clouds. For example, the morphology of an isolated SE cloud suggested formation of Kelvin-Helmholtz and Rayleigh-Taylor instabilities. Unfortunately, however, this SE cloud represents a rather late phase of shock - cloud interaction, involves a fairly dense preshock cloud precluding analysis of internal shock propagation, and has projection ambiguities; e.g. spectra now indicate that an initially suspected 'reverse shock' emission filament just west of the cloud is not that after all.

For testing the detailed hydrodynamical shocked cloud simulations that are now becoming available, ideally one would like to have the following: (a) a relatively isolated cloud with a low enough density to limit internal shock emission structure confusion due to superimposed cloud density variations, (b) an ISM cloud in a fairly early phase of shock interaction so that the complex interaction processes can be investigated right from the start as they first develop, and (c) a clear and simple interaction geometry with no 


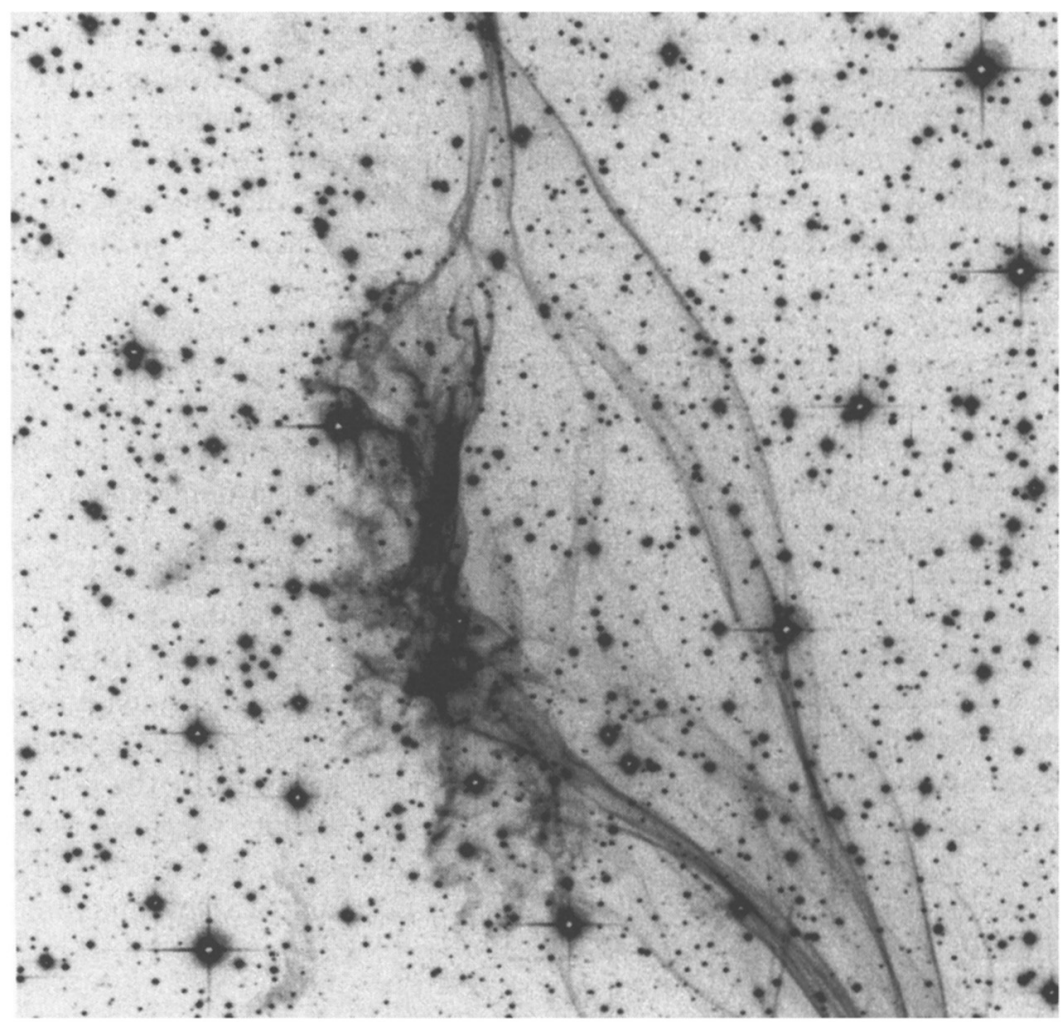

FIGURE 6. H $\alpha$ image of an isolated shocked emission cloud in the southwestern region of the Cygnus Loop. (From Downes \& Fesen 1993)

line-of-sight ambiguity as to location of the shock front or phase of shock-cloud collision. Does such an ideal shocked cloud exist in the Cygnus Loop? The answer appears to be yes.

A wide-field $\mathrm{H} \alpha$ reconnaissance of the entire Cygnus Loop recently uncovered a faint, isolated filamentary complex along the remnant's SW rim which offers an extraordinarily clear view of a single ISM cloud being engulfed by a high Mach shock wave (see Fig. 6). Distinct multiple radiative shock fronts can be seen in the cloud's interior with nonradiative fast shock emissions present along the cloud's sides and the undisturbed expanding shock front surface. Unlike any other filament regions so far studied in the Cygnus Loop, this cloud offers a particularly good view of an isolated cloud caught in the process of being overrun by the SNR's $200-400 \mathrm{~km} \mathrm{~s}^{-1}$ shock front. Spatial [O III] vs H $\alpha$ emission comparisons indicate a $2^{\prime \prime}-5^{\prime \prime}$ separation between nonradiative and radiative regions in the cloud suggestive of extended cooling and recombination postshock zones due to a low density around $1 \mathrm{~cm}^{-3}$. This also suggests a possible time dependent emission sequencing which is spread-out spatially many times larger than typically seen in SNRs. It is hoped that detailed spectral studies of this region may provide useful insights into the dynamical and line emission properties of shocked interstellar clouds. 


\section{REFERENCES}

Blair, W. P., Long, K. S., Vancura, O., Bowers, C. W., Conger, S., Davidsen, A. F., Kriss, G. A. \& Henry, R. B. C. 1992, ApJ, 399, 611 。

Chevalier, R. A. \& Kirshner, R. P. 1979, ApJ, 233, 154.

Downes, R. A. \& Fesen, R. A. 1993, in preparation.

Fesen, R. A. \& Becker, R. H. 1991, $A p J, 371,621$.

Fesen, R. A., Kwitter, K. B., \& Downes, R. A. 1992, AJ, 104, 719.

Fesen, R. A., Martin, C. L., \& Shull, J. M. 1992, ApJ, 399, 599.

Fesen, R. A. \& Staker, B. 1993, MNRAS, 263, 69.

Fesen, R. A., Wu, C.-C., Leventhal, M. \& Hamilton, A. J. S. 1988, ApJ, 327, 164.

Gunderson, K., \& Fesen, R. A. 1993, in preparation.

Henry, R. B. C. \& MacAlpine, G. 1982, ApJ, 258, 11.

Hester, J. J. 1987, $A p J$, 314, 187.

Klein, R. I., McKee, C. F. \& Colella, P. 1991, In Supernovae, ed. S. E. Woosley (Springer: New York) pp. 696.

MacAlpine, G. M., McGaugh, S. S., Mazzarella, J. M. \& Uomoto, A. 1989, ApJ, 342, 364.

MacAlpine, G. M., Lawrence, S. S., Uomoto, A., Lowenthal, J. D., Woodgate, B. E., Brown, L. W., \& Oliversen, R. J. 1993, ApJ, 432, L131

Michel, F. C., Scowen, P. A., Dufour, R. J. \& Hester, J. J. 1991, $A p J$, 368, 363.

Pequignot, D. \& Dennefeld, M. 1983, $A \& A, 120,249$.

Stone, J. M. \& Norman, M. L. 1992, $A p J, 390$, L17.

Uomoto, A \& MacAlpine, G. M. 1987, $A J, 931511$.

Wu, C.-C., Crenshaw, D. M., Fesen, R. A., Hamilton, A. J. S. \& Sarazin, C. L. 1993, ApJ, 416, 247. 